\title{
(6) OPEN ACCESS \\ Lipids, adiposity and tendinopathy: is there a mechanistic link? Critical review
}

\author{
Alex Scott, ${ }_{1}^{1,2}$ Johannes Zwerver, ${ }^{3}$ Navi Grewal, ${ }^{1,2}$ Agnetha de Sa, ${ }_{1}^{1,2}$ \\ Thuraya Alktebi, ${ }^{1,2}$ David I Granville, ${ }^{4}$ David A Hart ${ }^{5}$
}

${ }^{1}$ Department of Physical Therapy, University of British Columbia, Vancouver, British Columbia, Canada

${ }^{2}$ Vancouver Coastal Health Research Institute, Centre for Hip Health and Mobility, Vancouver, British Columbia, Canada

${ }^{3}$ University of Groningen, University Medical Center Groningen, Center for Sports Medicine, Groningen,

The Netherlands

${ }^{4}$ UBC James Hogg Research Centre, Institute for Heart + Lung Health, St. Paul's Hospital, University of British Columbia, Vancouver, Canada University of Calgary, McCaig Institute for Bone and Joint Health, Calgary, Alberta, Canada

\section{Correspondence to}

Dr Alex Scott, Department of Physical Therapy, University of British Columbia, Vancouver, 2177 Wesbrook Mall British Columbia, Canada V6T 1 Z3 ascott@interchange.ubc.ca

Accepted 15 November 2014 Published Online First

8 December 2014
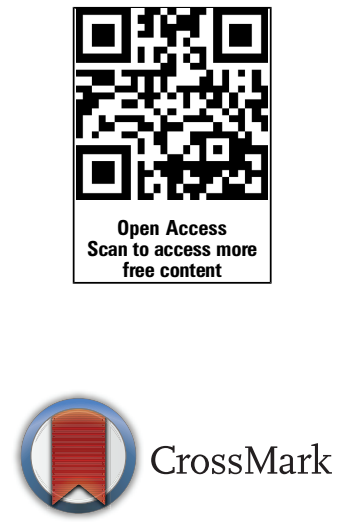

To cite: Scott A, Zwerver J, Grewal N, et al. Br J Sports Med 2015:49:984-988.

\begin{abstract}
Being overweight or obese is associated with an elevated risk of tendon pathology. However, for sportspeople the epidemiological data linking weight or adiposity on one hand, and risk of tendon pathology on the other, are less consistent. Indeed, the mechanistic links between diet, adiposity and tendon pathology remain largely unexamined. Recent studies have begun to examine the effects of dietary interventions on outcomes such as tendon biomechanics or pain. Oxidised low-density lipoprotein has been shown to (A) accumulate in the tendon tissues of mice that eat a fatty diet and (B) induce a pathological phenotype in human tendon cells. This paper addresses the current debate: is excessive body mass index (causing increased load and strain on tendon tissue) per se the underlying mechanism? Or do local or systemic influences of fat on tendons predispose to tendon pathology? This narrative review argues that excessive blood lipids may be an important avenue for clinical investigations.
\end{abstract}

\section{INTRODUCTION}

Minimising the impact of tendon pathology is an important clinical goal for those treating elite or recreational sportspeople. The same goal applies when prescribing exercise as medicine for weight reduction in sedentary individuals, who may be prone to acute or overuse injury when beginning a training programme.

Individualised load management strategies for patients undergoing rehabilitation for tendinopathy or tendon rupture (including identification and correction of biomechanical faults, and attention to training intensity and volume) are well recognised components of clinical practice. ${ }^{1}$ Although the load management strategy is not based on level 1 evidence, the approach emerged from clinical experience and close observation of patient characteristics associated with treatment success or failure. In a similar manner, Cook and co-workers ${ }^{2}$ reported their clinical impression that individuals with thicker waists were more likely to suffer from patellar tendinopathy. This impression was followed up with a small case-control study, which suggested that larger waist measurement may be a significant risk factor for patellar tendon pathology. ${ }^{3}$ This observation prompted further research into (A) the strength of this association in various populations that experience tendon pathology (including tendinopathy, tendon rupture and asymptomatic lesions identified on imaging) and (B) the underlying mechanism. This research can help the clinician decide whether adiposity needs to be accounted for as part of a holistic tendon injury management programme in active individuals (most of whom have body mass index (BMI) and lipid values within normal limits).

In this paper, we first examine key epidemiological studies that have been conducted in primarily sedentary populations. Although tendon pathology may present quite differently in the general population than in sportspeople, there appear to be some common themes. This is followed by a review of the available epidemiological evidence in sportspeople. Finally, we highlight potential mechanisms underlying the observed associations between adiposity and tendon pathology and identify some directions for future research.

\section{TERMINOLOGY}

Pathology may be defined as 'The anatomic or functional manifestations of a disease'. It may be symptomatic or asymptomatic. Tendon pain, rupture and abnormal imaging may share an underlying pathology (tendinosis), but this cannot be assumed in the absence of histological samples. ${ }^{4}$ In this paper, we therefore use the term 'tendon pathology' to refer to tendinopathy, rupture or asymptomatic lesions.

\section{EPIDEMIOLOGICAL EVIDENCE-POPULATION DATA}

One of the largest studies that examined the risk factors of tendon pathology was a 2014 casecontrol study conducted in the UK. ${ }^{5}$ Five thousand individuals in the general population who had received a diagnosis of rotator cuff disease (tendinopathy, bursitis, impingement, tears and calcific tendinopathy) were randomly selected from a health registry. This resulted in a sample with equal sex distribution (ie, gender was not a risk factor) and the patients were matched by age, sex and general practice with non-rotator cuff cases from the same registry. The average BMI of people with rotator cuff disease $\left(26.5 \mathrm{~kg} / \mathrm{m}^{2}\right)$ was significantly higher than that of the control group $\left(25.9 \mathrm{~kg} / \mathrm{m}^{2}, \mathrm{p}<0.01\right)$. Furthermore, the incidence of rotator cuff disease was higher in those who were overweight (BMI $25.1-30 \mathrm{~kg} / \mathrm{m}^{2}$ ) or obese (BMI $30.1-40 \mathrm{~kg} / \mathrm{m}^{2}$ ). BMI data were only available for $67 \%$ of those with tendon pathology and $61 \%$ of controls.

In this study, people who consulted a doctor more frequently (as the overweight and obese do $)^{6}$ were more likely to receive a diagnosis of rotator cuff disease. Is it the case that people who are overweight or obese are more likely to receive a diagnosis of tendon pathology because they are more frequently in contact with doctors? Or is it that they actually experience more tendon pathology, and are also in contact with doctors about other problems (in which case, controlling for 
consultation rate would result in an underestimation of the real OR for BMI).

Another large, well conducted case-control study (with slightly different methodology) was conducted in Finland and published in 2010, with similar findings to the UK study. ${ }^{7}$ In total, 6237 individuals participated in a structured interview and clinical examination. Rotator cuff tendinopathy (probable or possible) was identified in 175 participants (roughly equally divided among men and women) in a larger group of people with shoulder pain (approximately 1000). In men and women, increasing waist circumference was associated with increasing risk of rotator cuff tendinopathy. Type I diabetes was also associated with rotator cuff disease (in men, but not women). In keeping with the above studies, Wendelboe et $a l^{8}$ examined the association between BMI and those undergoing rotator cuff repair surgery $(n=311)$ compared with controls $(n=933)$. This study identified a dose-response in men and women, with increasing BMI associated with increasing OR of rotator cuff repair, up to 3 in obese females $\left(\mathrm{BMI}>30 \mathrm{~kg} / \mathrm{m}^{2}\right)$. As with the above studies, because this was a case-control study it is not possible to conclude causality (ie, it is possible that a shoulder injury results in reduced activity and therefore increasing BMI). However, the existence of a dose-response argues strongly against the latter explanation.

Another large, UK population-based study examined risk factors for lateral epicondylalgia in the general community. Almost 5000 individuals were matched by age, sex and general practice with controls. BMI data were available for $69 \%$ of individuals with lateral epicondylalgia and $61 \%$ of controls. As in the previous study by the same group, obesity was identified as a significant risk factor, but this disappeared when controlling for consultation rate. ${ }^{9}$ A longitudinal study also examined, among other variables, the risk of developing lateral epicondylalgia in 1107 workers engaged in manual labour who were categorised as obese or non-obese. Obesity led to an OR of 2.4 (95\% CI 1.2 to 4.8 ) for developing lateral epicondylalgia in this study. ${ }^{10}$ Shiri et $a l^{11}$ also reported that in a study of 4783 Finnish individuals, of whom 168 had medial or lateral epicondylitis, there was a higher incidence of medial (but not lateral) epicondylalgia among individuals in the highest waist-hip ratio category $(>0.95$ : $\mathrm{OR}=3.5 ; 95 \%$ CI 1.6 to 7.5$)$.

The evidence for an association between adiposity and tendon pathology at other tendon locations is preliminary, but generally supports a relation between BMI and increasing risk of pathology for some, but not all tendons. ${ }^{12}{ }^{13}$ Increased BMI was not identified as a risk factor in a small $(n=22)$ case-control study of pes anserine syndrome, however, diagnostic imaging to confirm the presence or absence of tendinopathy was not reported. ${ }^{14}$ In 81 healthy individuals who were either runners or non-runners with no history of Achilles tendon pain, sonographic abnormalities (indicating the presence of asymptomatic tendon pathology) were more frequently observed in the Achilles tendons of overweight participants (24\% vs $8 \%) .{ }^{15}$ In this study, the Achilles tendons of overweight sedentary individuals were thicker than the tendons of normal weight sedentary individuals (5.2 vs $4.4 \mathrm{~mm}, \mathrm{p}=0.002)$, and overweight runners with sonographic alterations had a higher body weight than overweight runners without sonographic abnormalities. Frey and Zamora ${ }^{16}$ surveyed 1411 patients in an orthopaedic foot and ankle clinic and found that a BMI $>25 \mathrm{~kg} / \mathrm{m}^{2}$ significantly increased the risk (OR 1.9, 95\% CI 1.39 to 2.66) of tendinopathy (Achilles, posterior tibal or peroneal). Similar findings, but specifically with regard to the increased risk of Achilles tendinopathy in overweight individuals, were reported in two other case-control studies. ${ }^{17} 18$ Finally, MRI evidence of patellar tendon pathology is associated with a history of elevated BMI or elevated body weight. ${ }^{19}$

\section{EPIDEMIOLOGICAL EVIDENCE-SPORTSPEOPLE}

Epidemiological evidence among sportspeople is scarcer than that for the general population. A Union of European Football Associations (UEFA) study tracked Achilles tendon pathology (tendinopathy or rupture) among 1743 male professional footballers. There was no difference in BMI between those who did or did not experience an Achilles tendon injury (23.6 \pm 1.3 vs $23.4 \pm 1.4 \mathrm{~kg} / \mathrm{m}^{2}$ respectively, $\mathrm{p}=0.092$ ), although waist measurements were not reported. ${ }^{20}$ The findings were similar in two smaller studies in elite-level volleyball players ${ }^{3}{ }^{21}$ in that BMI was not related to the presence of sonographic patellar tendon pathology or to the development of symptomatic tendinopathy. However, in the former study, waist circumference was measured, and was a significant risk factor: those with a waist circumference of $83 \mathrm{~cm}$ or greater had a 74\% chance of patellar tendon pathology, compared with $15 \%$ in those with $<83 \mathrm{~cm}$ waistline. The authors speculated that, in sportspeople, waist circumference may be a better predictor of tendon pathology than BMI per se, ${ }^{3}$ but this finding was not confirmed by Visnes and Bahr. $^{21}$

\section{EPIDEMIOLOGICAL EVIDENCE-SYSTEMATIC REVIEW AND META-ANALYSIS}

Gaida et $a l^{12}$ conducted a systematic review of observational studies and included all studies that compared adiposity between individuals with or without tendon pathology. Twenty-eight studies including 19949 individuals were analysed. The included population was extremely diverse, including asymptomatic cases (defined by imaging), as well as tendinopathy and rupture cases. The authors concluded that elevated adiposity is significantly associated with tendon pathology. Indeed, $81 \%$ of studies that recruited patients with tendon pathology (ie, people presenting to a healthcare practitioner for management of tendon pain) indicated a positive association of tendon pathology with adiposity. The authors recommended further research to determine whether interventions to reduce adiposity would improve the results of treatment or injury prevention.

\section{EVIDENCE REGARDING MECHANICAL LOAD AS A MECHANISM LINKING ADIPOSITY AND TENDON PATHOLOGY}

Does increased body weight predispose to pathology because sportspeople subject their tendons to high or, arguably, excessive loads? ${ }^{22}$ There is limited evidence to address this question for upper as well as lower extremity tendons. However, the anatomical and functional differences between upper and lower limb tendons make it difficult to determine the importance of similarities and differences in the data across population and athletic cohorts.

Garner et $a l^{23}$ examined the impact of body composition on the magnitude moment arms at the shoulder and elbow during throwing. There was a significant relationship between increasing fat content in the arm, and increasing magnitude of injury-related throwing mechanics. Given that the shoulder tendons are frequently injured in sportspeople who throw, a case-control study in a larger population could shed further light on this potential mechanism of injury. One could argue that a heavier arm leads to higher loads through the rotator cuff tendons, however, this has not been directly demonstrated and further research would be required to confirm or refute this hypothesis. It would also be of interest to examine injury-related 
mechanics in a variety of other tasks of relevance to a sedentary population (eg, lifting, reaching). Gupta et $a l^{24}$ compared the scapular kinematics in individuals of high $\left(>27 \mathrm{~kg} / \mathrm{m}^{2}\right)$ or low $\left(<23 \mathrm{~kg} / \mathrm{m}^{2}\right)$ BMI in 20 individuals. Those with higher BMI demonstrated altered scapular kinematics (increased scapular upward rotation), which was suggested to be a strategy to compensate for increased arm mass.

With regard to the lower extremity, Wearing et $a l^{25}$ examined the structure (ultrasound imaging) and biomechanical response of the Achilles tendons in individuals of low $\left(<23 \mathrm{~kg} / \mathrm{m}^{2}\right)$ or high $\left(>25 \mathrm{~kg} / \mathrm{m}^{2}\right)$ BMI. The Achilles tendons of the high BMI group were on average $12 \%$ thicker, and this figure was used by the authors to estimate that the cross-sectional area (CSA) of the Achilles tendon was a remarkable $38 \%$ larger for overweight individuals. This increased CSA was further estimated to be capable of offsetting (compensating for) the increased tendon load that results from increased body weight. ${ }^{25}$ In other words, increased body weight may act as an adaptive stimulus capable of increasing tendon CSA, as has been shown with resistance training programmes. ${ }^{26}$ However, the transverse strains of the Achilles tendon were reduced in those with elevated BMI, suggesting that the collagenous matrix was not only larger, but abnormal in structure or composition. Therefore, the observed increase in tendon CSA in the individuals with high BMI may represent a pathological as opposed to adaptive response. However, it is unclear whether this response is due to increased load through the tendon (due to increased body weight), or due to a systemic influence.

When interpreting the findings of epidemiological studies, one has to remember that the OR represents a statistical association, not necessarily a causal one. Thus, increased risk for development of tendon pathology with increased BMI or waist girth indicates that the variables (tendon pathology and BMI) are associated, despite the multitude of other genetic and nongenetic risk factors. The epidemiological assessments discussed above provide some impetus to further focus investigations into how the risk is manifested and why it occurs.

\section{EVIDENCE FOR A SYSTEMIC LINK BETWEEN ADIPOSITY AND TENDON PATHOLOGY-ARE BLOOD LIPIDS THE MISSING LINK?}

Given that the case-control studies reviewed above have generally been consistent in identifying that the increased BMI or waist circumference/waist-hip ratio are risk factors for tendon pathology, it is reasonable to ask whether there may be an 'upstream' systemic influence of excessive blood lipid. Indeed, inherited hyperlipidaemia is a well-known cause of tendon pathology, ${ }^{27}$ and it is also possible that diet-related or age-related hyperlipidaemia, or mildly elevated total cholesterol, ${ }^{28}$ may predispose to tendon pathology in otherwise healthy individuals.

Total cholesterol levels within the blood stream are influenced by diet, and by the efficiency with which cholesterol is transported among tissues by cholesterol-rich lipoproteins. Increases in lowdensity lipoprotein (LDL) or decreases in high-density lipoprotein (HDL) are associated with the accumulation of cholesterol in nonliver tissues. ${ }^{29}$ LDLs are primarily responsible for transporting cholesterol between the liver and the circulation, and HDLs are responsible for returning cholesterol to the liver. A key stage in the initiation and progression of atherosclerosis, which has recently come into the spotlight, is the oxidation of LDL. LDL oxidation is thought to occur in the extravascular tissue spaces, which are less protected by antioxidants than in the plasma. Oxidised LDL (oxLDL) has pathogenic effects on a variety of cell types including smooth muscle cells, macrophages and endothelial cells. ${ }^{30}$ OxLDL levels are elevated in the obese and because of its pathogenic role it has been proposed as a potential marker for atherosclerosis risk or severity. The liver is reported to be responsible for the removal of oxLDL from the circulation.

Mathiak et $a l^{31}$ measured the total cholesterol level of 31/41 patients (average age 40.2 years, range 24-78) who had undergone surgical repair of the ruptured Achilles tendon. Remarkably, $83 \%$ of those sampled had elevated total cholesterol levels $(>200 \mathrm{mg} / \mathrm{dL})$ and were referred for further medical management. However, these findings are in contrast with a study by Haacke and Parwaresch, ${ }^{32}$ who measured lipid levels in 59 patients with Achilles tendon rupture. In this study, only $15 \%$ of those sampled (average age 32 years, range 15-42) had increased total cholesterol levels. In those with increased cholesterol, histopathology demonstrated evidence of lipid accumulation within the Achilles tendons; this finding was not present in the 50 cases with normal cholesterol levels, although the blinding and observation procedures were not detailed. ${ }^{32}$ Recent findings by Ozgurtas $e t a l^{33}$ are supportive of observations by Mathiak et al, and this study had the advantage of including a control group (figure 1). Of the 47 Achilles tendon rupture cases, 74.5\% demonstrated elevated total cholesterol and 70.2\% had elevated LDL, compared to $25.5 \%$ and $29.8 \%$ in controls, respectively. The blood sampling varied between the case and control groups; the patient group was sampled $6-8 \mathrm{~h}$ following rupture, while the control group was fasting. However, fasting before lipid testing has been shown to result in less than $2 \%$ variation for total cholesterol and less than $10 \%$ for LDL. ${ }^{34}$ Thus, preliminary data suggest that elevated total cholesterol or LDL may be a more frequent finding among patients with Achilles tendinopathy compared with controls, however, definitive data are not yet available.

Two studies of the rotator cuff present further preliminary data on this potential link between blood lipids and tendon pathology. Longo $e t a l^{35}$ measured serum triglycerides and total cholesterol in 240 patients, 120 of whom were undergoing arthroscopic rotator cuff repair and 120 of whom were controls (patients undergoing meniscectomy). In the rotator cuff
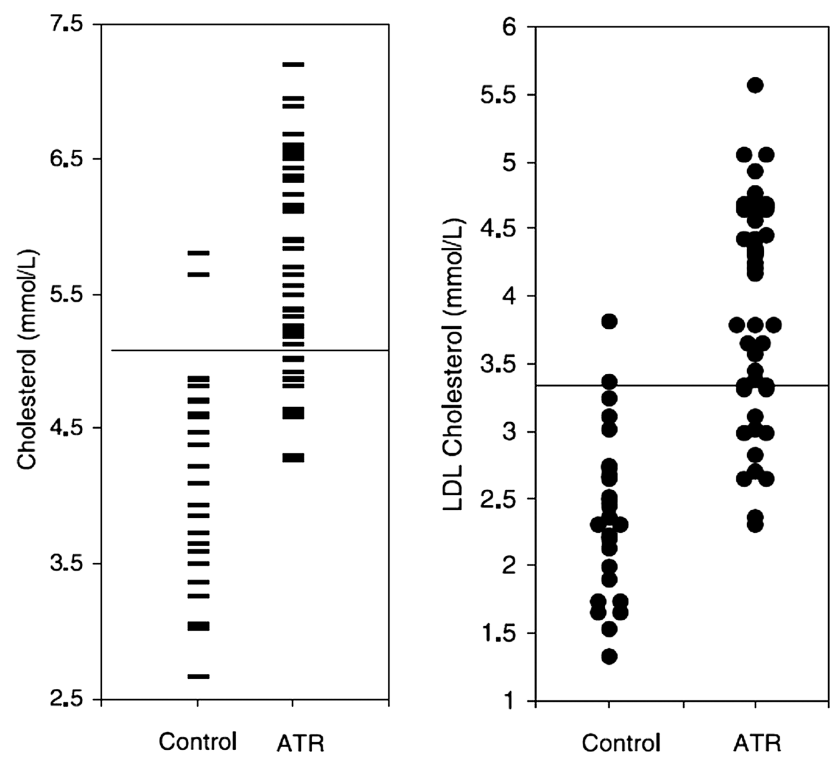

Figure 1 Lipid results in patients with Achilles tendon rupture (ATR) versus case-controls (LDL, low-density lipoprotein). ${ }^{33}$ Please see original reference for other significant differences in blood lipid levels between control and ATR. With permission of Elsevier. 
patients, $69 \%$ of patients had elevated total cholesterol ( $>5.2 \mathrm{mmol} / \mathrm{L}$ ) compared with $66 \%$ of those undergoing meniscectomy. In contrast, Abboud et $a l^{36}$ compared 74 rotator cuff surgical candidates with non-cuff shoulder pain patients and found $63 \%$ of those with rotator cuff problems had elevated total cholesterol $(>240 \mathrm{mg} / \mathrm{dL})$ compared with $28 \%$ in the control group.

With regard to non-surgical cases of tendinopathy, the results are also preliminary. Gaida et $a l^{28}$ measured serum lipids in 60 members of the general population with chronic painful midportion Achilles tendinopathy and in 60 age-matched and BMI-matched controls, and found evidence of underlying dislipidemia including a significant elevation of triglyceride levels. In a population-based study of 5871 Finnish people, Shiri et al ${ }^{11}$ found no significant difference in LDL or HDL levels among those with or without chronic lateral or medial epicondylitis.

\section{EVIDENCE FOR A LINK BETWEEN BLOOD GLUCOSE AND TENDON RUPTURE OR TENDINOPATHY?}

Elevated blood glucose levels are well known to have a profound influence on tendon health, and this topic has recently been reviewed elsewhere. ${ }^{37}$ Certainly, the link between diagnosed diabetes mellitus and poor tendon health is well established. One could ask whether blood glucose levels in the prediabetic overweight individual could already predispose to tendon injury, and whether this may be another mechanism behind the association of BMI and tendon pathology. This question has not been addressed to our knowledge. Arguing against this line of reasoning, in the large population-based studies cited above, BMI was a stronger risk factor for tendinopathy than a diagnosis of diabetes mellitus.

\section{EFFECT OF DIETARY INTERVENTIONS TO MITIGATE/ PREVENT TENDINOPATHY}

Given that case-control studies have identified that increased BMI or waist circumference and elevated blood lipids are risk factors for tendon pathology, it is reasonable to ask whether an intervention to reduce these would have any effect on the risk of developing tendinopathy or tendon rupture. To the best of our knowledge, this question has hardly been addressed. A recent study in mice demonstrated that mice that were fed a high-fat diet experienced a drop in Achilles tendon modulus compared with mice on a normal diet, and neither exercise nor administration of a nutritional supplement (branched chain amino acid) were able to mitigate this decline in biomechanical function. ${ }^{38}$ Szczurko et $a l^{39}$ conducted a patient-blinded and assessor-blinded randomised controlled trial to examine the impact of a naturopathic intervention on rotator cuff tendinopathy; the intervention included individualised dietary counselling, acupuncture and prescribing of a natural supplement (phlogenzym). Patients who received the naturopathic intervention experienced a greater improvement in shoulder function than those who received placebo or range of motion exercises. The impact of the dietary intervention in this clinical trial is difficult to gauge, and the exercises used in the trial are not consistent with those with proven clinical efficacy. ${ }^{40}$

\section{EFFECT OF STATINS AND DEVELOPMENT OF TENDON PATHOLOGY}

Statins are a class of drugs designed to treat hyperlipidaemia by inhibiting the enzyme HMG-coenzyme A reductase (discussed in ref. 41). While effective in many patients with elevated plasma lipids, they are not without their side effects (discussed in refs. 41 and 42). Individuals with familial hyperlipidaemias are at risk for tendon xanthomas (discussed in refs. 27 and 42), which can impair function. When placed on statins, tendon thickening and the xanthomas are reported to regress. ${ }^{27} 4344$ However, a small subset of patients treated with statins has been reported to develop tendinopathies or tendon ruptures. ${ }^{41} 4245-47$

The mechanism(s) by which statins enhance the risk for tendon ruptures in this subset of patients is not known. However, recently de Oliveira et $a l^{41}$ described biochemical changes in the Achilles tendons of rats chronically treated with statins. They report that in the rat studies, an imbalance between catabolic and anabolic activities in the treated tendons was detected. Thus, alterations in metabolism (eg, increased MMP expression and altered matrix expression) were associated with statin treatment.

Such findings raise two important points: (1) questions regarding drug use, particularly of statins, should be included in all clinical and epidemiological studies of tendinopathy and (2) further studies on the influence of statins on tendon repair after injury (eg, tendinopathy, surgery, rupture) should be undertaken to better understand the basis for risk associated with drug treatment.

\section{INTEGRATED MODEL-THE SYSTEMIC INFLUENCE OF LIPID ON TENDONS AND INFLUENCE ON BIOMECHANICAL FUNCTION}

We recently conducted a study on mice to examine the influences of localised lipid (within or around the tendon tissue) versus systemic lipid (hypercholesterolaemia) on tendon biomechanical properties. ${ }^{48}$ Two strains of mice (normal mice or mice predisposed to developing hypercholesterolaemia, the apolipoprotein E knockout (ApoE-KO) model) were fed a normal diet, or a high-fat diet. Interestingly, the accumulation of adipose tissue around tendons occurred in normal mice that were fed a high-fat diet, and their tendons demonstrated a significant reduction in failure load and stress compared with mice on a normal diet. However, an even larger reduction in failure load and stress occurred in the tendons of ApoE-KO mice (particularly when combined with a high-fat diet), even though these mice (A) did not accumulate significant amounts of peritendinous adipose tissue and (B) gained less weight than their normal counterparts. This constellation of findings argues in favour of a systemic, rather than local, influence of dietary lipid or its byproducts on tendon health. This finding is in keeping with data demonstrating impaired tendon healing in a high-fat diet-induced mouse model of obesity and type 2 diabetes. ${ }^{49}$ Furthermore, when we applied oxLDL at physiological levels to human tenocytes, gene expression was altered-the tenocytes adopted an abnormal, proliferative behaviour, reducing their collagen I expression and increasing MMP expression.

Taken together, we propose the following (figure 2). Excessive dietary lipid can do either (or both) of the following: (1) cause accumulation of body weight and/or abdominal adiposity, which places excessive mechanical load on tendon tissues or (2) cause dislipidemia, which exerts a systemic biological effect on tenocytes, thereby weakening the tendons. In the sedentary population more at risk of increasing BMI, either or both mechanisms may be dominant. Excessive load through a tendon previously weakened by metabolically disturbed tenocytes could predispose to injury. In athletic populations with normal (or low) body weight, the second mechanism may play more of a role. Both mechanisms could also interact with all of the other known biomechanical and non-biomechanical risk factors already identified for tendon pathology, such as age, hormonal status, genetics and others. ${ }^{50-52}$ 
Normal diet
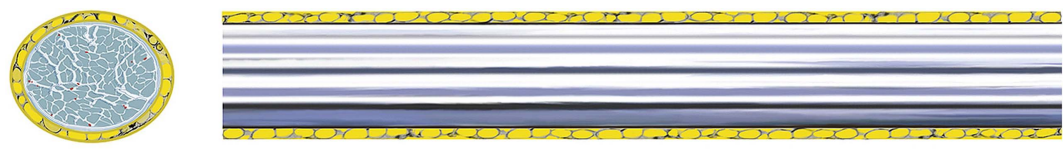

High-fat diet, Normal BMI
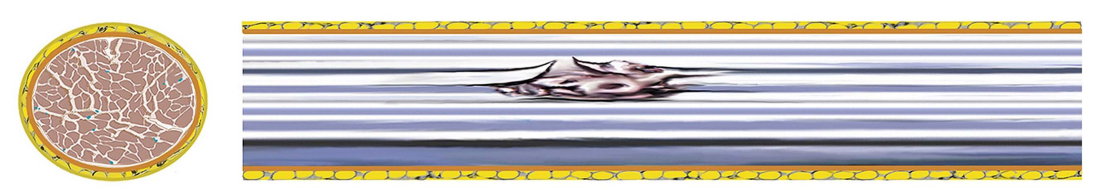

High fat diet, High BMI
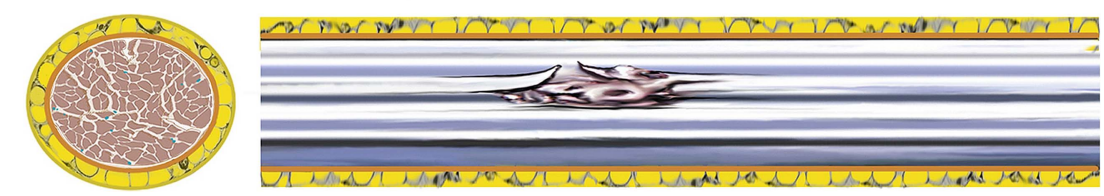

Figure 2 Short (left) and long (right) axis views through hypothetical tendons from three individuals. Top: healthy individual with normal body mass index (BMI) and healthy diet, and no cholesterol accumulation in the collagen fibres (healthy, denoted as bluish). Middle: athlete consuming a high-fat diet. The tendon is slightly larger (adaptation to loading), BMI is normal (due to caloric expenditure), but the consumption of fatty diet has led to accumulation of cholesterol (oxidised low-density lipoprotein (oxLDL, brown) with resulting weakening and predisposition to microinjury or rupture. Bottom: recreational, overweight athlete. The tendon demonstrates increased peritendinous adipose tissue as well as increased cholesterol accumulation (oxLDL, brown). The increased body mass of the individual may exacerbate the risk of developing tendon pathology, and a high-fat diet may delay healing.

\section{CONCLUSION}

The epidemiology of tendon injuries (ruptures and tendinopathy) has highlighted the association between BMI or adiposity and the risk of pathology. A potential role of dislipidemia in predisposing to tendon pathology has been identified in the general population. Animal studies suggest that dislipidemia may directly and negatively influence tendons, but this mechanism needs to be confirmed in humans.

Given that exercise is a common prescription for overweight individuals, the increased risk of tendon injury in this patient group could be an impediment to healthy lifestyle change. In the future, larger scale epidemiological studies in a variety of sedentary and athletic populations could account better and more specifically for the identified potential risk factors discussed in this paper (adiposity, diet, blood lipids) to confirm or refute the hypotheses presented here and, in the future, lead to holistic exercise prescriptions with consideration for the underlying health of musculotendinous tissues.

\section{What are the new findings?}

- Large epidemiological studies in the general population support the existence of a relationship between body mass index (or body weight or waist circumference) and the occurrence of tendon pathology, but this relation has not been observed in more elite athletic groups.

- There is evidence for a mechanical effect (increased load) and a systemic effect (eg, effects of circulating lipids), but the relative contributions of these two mechanisms remains preliminary and hypothetical.

- Data from animal studies suggest that a high-fat diet negatively influences tendon health.
Correction notice This article has been changed to review, and made Open Access since published Online First.

Contributors All authors contributed to writing and editing the manuscript. AS conceived the paper and wrote the first draft. The other authors made further written contributions and editorial suggestions.

\section{Competing interests None.}

Provenance and peer review Not commissioned; externally peer reviewed.

Open Access This is an Open Access article distributed in accordance with the Creative Commons Attribution Non Commercial (CC BY-NC 4.0) license, which permits others to distribute, remix, adapt, build upon this work non-commercially, and license their derivative works on different terms, provided the original work is properly cited and the use is non-commercial. See: http://creativecommons.org/ licenses/by-nc/4.0/

\section{REFERENCES}

1 Cook JL, Purdam CR. The challenge of managing tendinopathy in competing athletes. Br J Sports Med 2014;48:506-9.

2 Gaida JE, Cook JL, Bass SL. Adiposity and tendinopathy. Disabil Rehabil 2008;30:1555-62.

3 Malliaras P, Cook JL, Kent PM. Anthropometric risk factors for patellar tendon injury among volleyball players. Br J Sports Med 2007;41:259-63; discussion 63.

4 Rees JD, Stride M, Scott A. Tendons-time to revisit inflammation. Br J Sports Med 2014:48:1553-7

5 Titchener AG, White JJ, Hinchliffe SR, et al. Comorbidities in rotator cuff disease: a case-control study. J Shoulder Elbow Surg 2014;23:1282-8.

6 Pappa E, Kontodimopoulos N, Papadopoulos AA, et al. Physician consultations according to different BMI levels of the Greek general population. Int J Environ Res Public Health 2011:8:4300-11.

7 Rechardt M, Shiri R, Karppinen J, et al. Lifestyle and metabolic factors in relation to shoulder pain and rotator cuff tendinitis: a population-based study. BMC Musculoskelet Disord 2010;11:165.

8 Wendelboe AM, Hegmann KT, Gren LH, et al. Associations between body-mass index and surgery for rotator cuff tendinitis. J Bone Joint Surg Am 2004;86-A:743-7

9 Titchener AG, Fakis A, Tambe AA, et al. Risk factors in lateral epicondylitis (tennis elbow): a case-control study. J Hand Surg Eur Vol 2013;38:159-64.

10 Descatha A, Dale AM, Jaegers $L$, et al. Self-reported physical exposure association with medial and lateral epicondylitis incidence in a large longitudinal study. Occup Environ Med 2013;70:670-3. 
11 Shiri R, Viikari-Juntura $\mathrm{E}$, Varonen $\mathrm{H}$, et al. Prevalence and determinants of lateral and medial epicondylitis: a population study. Am J Epidemiol 2006;164:1065-74.

12 Gaida JE, Ashe MC, Bass SL, et al. Is adiposity an under-recognized risk factor for tendinopathy? A systematic review. Arthritis Rheum 2009;61:840-9.

13 Butterworth PA, Landorf KB, Smith SE, et al. The association between body mass index and musculoskeletal foot disorders: a systematic review. Obes Rev 2012;13:630-42.

14 Alvarez-Nemegyei J. Risk factors for pes anserinus tendinitis/bursitis syndrome: a case control study. J Clin Rheumatol 2007;13:63-5.

15 Abate M, Oliva F, Schiavone $C$, et al. Achilles tendinopathy in amateur runners: role of adiposity (tendinopathies and obesity). Muscles Ligaments Tendons $J$ 2012:2:44-8.

16 Frey C, Zamora J. The effects of obesity on orthopaedic foot and ankle pathology. Foot Ankle Int 2007;28:996-9.

17 Klein EE, Weil L Jr, Weil LS Sr, et al. Body mass index and Achilles tendonitis: a 10-year retrospective analysis. Foot Ankle Spec 2013;6:276-82.

18 Scott RT, Hyer CF, Granata A. The correlation of Achilles tendinopathy and body mass index. Foot Ankle Spec 2013;6:283-5.

19 Fairley J, Toppi J, Cicuttini F, et al. Association between obesity and magnetic resonance imaging defined patellar tendinopathy in community-based adults: a cross-sectional study. BMC Musculoskelet Disord 2014;15:266.

20 Gajhede-Knudsen M, Ekstrand J, Magnusson H, et al. Recurrence of Achilles tendon injuries in elite male football players is more common after early return to play: an 11-year follow-up of the UEFA Champions League injury study. $\mathrm{Br}$ J Sports Med 2013;47:763-8.

21 Visnes H, Bahr R. Training volume and body composition as risk factors for developing jumper's knee among young elite volleyball players. Scand J Med Sci Sports 2013:23:607-13.

22 Bahr MA, Bahr R. Jump frequency may contribute to risk of jumper's knee: a study of interindividual and sex differences in a total of 11,943 jumps video recorded during training and matches in young elite volleyball players. Br J Sports Med 2014:48:1322-6

23 Garner JC, MacDonald C, Wade C, et al. The influence of body composition on youth throwing kinetics. Pediatr Exerc Sci 2011;23:379-87.

24 Gupta M, Dashottar A, Borstad JD. Scapula kinematics differ by body mass index. J App/ Biomech 2013;29:380-5.

25 Wearing SC, Hooper SL, Grigg NL, et al. Overweight and obesity alters the cumulative transverse strain in the Achilles tendon immediately following exercise. J Bodyw Mov Ther 2013;17:316-21.

26 Kongsgaard M, Qvortrup K, Larsen J, et al. Fibril morphology and tendon mechanical properties in patellar tendinopathy: effects of heavy slow resistance training. Am J Sports Med 2010;38:749-56.

27 Tsouli SG, Kiortsis DN, Argyropoulou MI, et al. Pathogenesis, detection and treatment of Achilles tendon xanthomas. Eur J Clin Invest 2005;35:236-44.

28 Gaida JE, Alfredson L, Kiss ZS, et al. Dyslipidemia in Achilles tendinopathy is characteristic of insulin resistance. Med Sci Sports Exerc 2009;41:1194-7.

29 Babiak J, Rudel LL. Lipoproteins and atherosclerosis. Baillieres Clin Endocrinol Metab 1987:1:515-50.

30 Ishigaki Y, Oka Y, Katagiri H. Circulating oxidized LDL: a biomarker and a pathogenic factor. Curr Opin Lipidol 2009;20:363-9.

31 Mathiak G, Wening JV, Mathiak $M$, et al. Serum cholesterol is elevated in patients with Achilles tendon ruptures. Arch Orthop Trauma Surg 1999;119:280-4.
32 Haacke H, Parwaresch MR. Spontaneous rupture of the Achilles tendon-a sign of hyperlipoproteinaemia (HLP) type II. Klin Wochenschr 1979;57:397-400.

33 Ozgurtas T, Yildiz C, Serdar M, et al. Is high concentration of serum lipids a risk factor for Achilles tendon rupture? Clin Chim Acta 2003;331:25-8.

34 Sidhu D, Naugler C. Fasting time and lipid levels in a community-based population: a cross-sectional study. Arch Intern Med 2012;172:1707-10.

35 Longo UG, Franceschi F, Spiezia F, et al. Triglycerides and total serum cholesterol in rotator cuff tears: do they matter? Br J Sports Med 2010;44:948-51.

36 Abboud JA, Kim JS. The effect of hypercholesterolemia on rotator cuff disease. Clin Orthop Rel Res 2010;468:1493-7.

37 Abate M, Schiavone C, Salini V, et al. Occurrence of tendon pathologies in metabolic disorders. Rheumatology (Oxford) 2013;52:599-608.

38 Boivin GP, Platt KM, Corbett J, et al. The effects of high-fat diet, branched-chainamino acids and exercise on female C57BL/6 mouse Achilles tendon biomechanical properties. Bone Joint Res 2013;2:186-92.

39 Szczurko 0, Cooley K, Mills EJ, et al. Naturopathic treatment of rotator cuff tendinitis among Canadian postal workers: a randomized controlled trial. Arthritis Rheum 2009;61:1037-45.

40 Holmgren $T$, Hallgren HB, Oberg B, et al. Effect of specific exercise strategy on need for surgery in patients with subacromial impingement syndrome: randomised controlled study. Br J Sports Med 2014;48:1456-7.

41 de Oliveira LP, Vieira CP, Da Re Guerra F, et al. Statins induce biochemical changes in the Achilles tendon after chronic treatment. Toxicology 2013;311:162-8.

42 Esenkaya I, Unay K. Tendon, tendon healing, hyperlipidemia and statins. Muscles Ligaments Tendons J 2011;1:169-71.

43 Kolovou G, Daskalova D, Mastorakou I, et al. Regression of Achilles tendon xanthomas evaluated by CT scan after hypolipidemic treatment with simvastatin. A case report. Angiology 2004;55:335-9.

44 Ahn JH, Chun TJ, Lee S. Nodular excision for painful localized Achilles tendon xanthomas in type II hyperlipoproteinemia: a case report. J Foot Ankle Surg 2011;50:603-6.

45 Carmont MR, Highland AM, Blundell CM, et al. Simultaneous bilateral Achilles tendon ruptures associated with statin medication despite regular rock climbing exercise. Phys Ther Sport 2009;10:150-2.

46 Celik EC, Ozbaydar M, Ofluoglu D, et al. Simultaneous and spontaneous bilateral quadriceps tendons rupture. Am J Phys Med Rehabil 2012;91:631-4.

47 Marie I, Delafenetre H, Massy N, et al. Tendinous disorders attributed to statins: a study on ninety-six spontaneous reports in the period 1990-2005 and review of the literature. Arthritis Rheum 2008;59:367-72.

48 Grewal N, Thornton GM, Behzad H, et al. Accumulation of oxidized LDL in the tendon tissues of C57BL/6 or Apolipoprotein E knock-out mice that consume a high fat diet: potential impact on tendon health. PLOS ONE 2014;9:e114214.

49 David MA, Jones KH, Inzana JA, et al. Tendon repair is compromised in a high fat diet-induced mouse model of obesity and type 2 diabetes. PLOS ONE 2014;9: e91234.

50 van der Worp $\mathrm{H}$, van Ark M, Roerink S, et al. Risk factors for patellar tendinopathy: a systematic review of the literature. Br J Sports Med 2011;45:446-52.

51 Maffulli N, Wong J, Almekinders LC. Types and epidemiology of tendinopathy. Clin Sports Med 2003;22:675-92.

52 Longo UG, Berton A, Papapietro N, et al. Epidemiology, genetics and biological factors of rotator cuff tears. Med Sport Sci 2012;57:1-9. 